\title{
Peptide-based NTA(Ni)-nanodiscs for studying membrane enhanced FGFR1 kinase activities
}

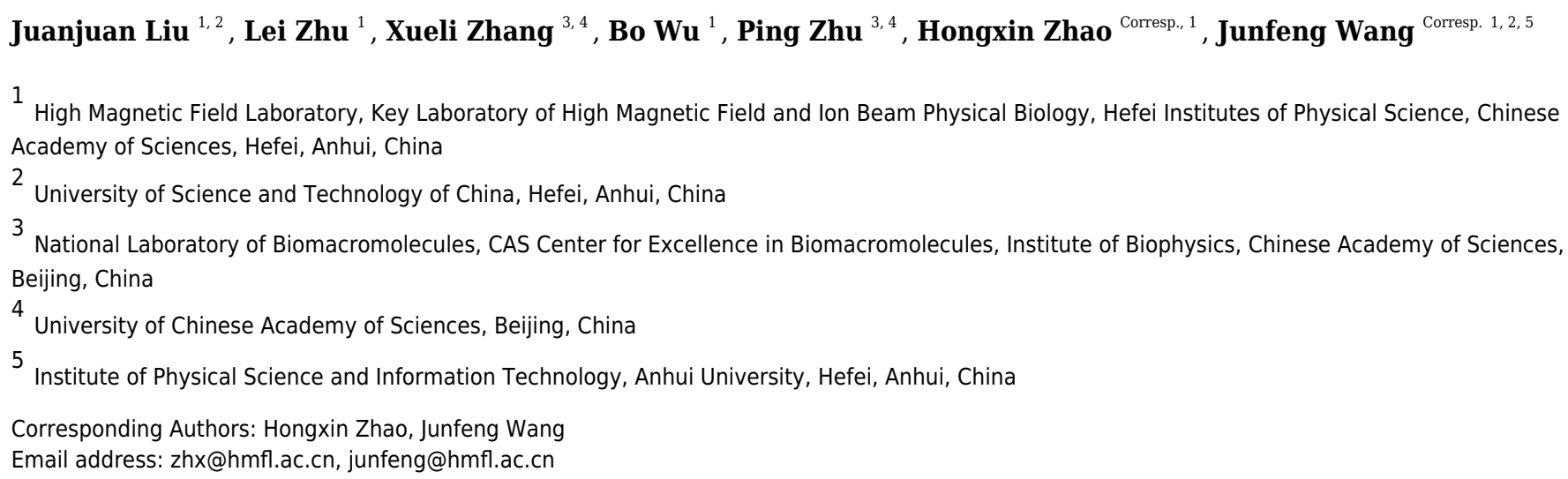

Tyrosine autophosphorylation plays a crucial regulatory role in the kinase activities of fibroblast growth factor receptors (FGFRs), and in the recruitment and activation of downstream intracellular signaling pathways. Biophysical and biochemical investigations of FGFR kinase domains in membrane environments offer key insights into phosphorylation mechanisms. Hence, we constructed nickel chelating nanodiscs based on a 22-residue peptide. The spontaneous anchoring of $\mathrm{N}$-terminal $\mathrm{His}_{6}$-tagged FGFR1c kinase domain (FGFR1K) onto peptide nanodiscs grants FGFR1K orientations occurring on native plasma membranes. Following membrane incorporation, the autophosphorylation of FGFR1K, as exemplified by Y653 and Y654 in the A-loop and the total tyrosine phosphorylation, increase significantly. This in vitro reconstitution system may be applicable to studies of other membrane associated phenomena. 


\section{Peptide-based NTA(Ni)-nanodiscs for studying membrane} 2 enhanced FGFR1 kinase activities

3 Juanjuan Liu ${ }^{1,2}$, Lei Zhu ${ }^{1}$, Xueli Zhang ${ }^{3,4}$, Bo Wu ${ }^{1}$, Ping Zhu ${ }^{3,4}$, Hongxin Zhao ${ }^{1}$ and Junfeng

4 Wang ${ }^{1,2,5}$

$5 \quad{ }^{1}$ High Magnetic Field Laboratory, Key Laboratory of High Magnetic Field and Ion Beam

6 Physical Biology, Hefei Institutes of Physical Science, Chinese Academy of Sciences, Hefei

7 230031, Anhui, P. R. China.

$8 \quad{ }^{2}$ University of Science and Technology of China, Hefei 230036, Anhui, P. R. China.

$9 \quad{ }^{3}$ National Laboratory of Biomacromolecules, CAS Center for Excellence in Biomacromolecules, Institute of Biophysics, Chinese Academy of Sciences, Beijing 100101, China.

$11{ }^{4}$ University of Chinese Academy of Sciences, Beijing 100049, China.

$12{ }^{5}$ Institute of Physical Science and Information Technology, Anhui University, Hefei 230031, 13 Anhui, P. R. China.

14 Corresponding Author:

15 Hongxin Zhao ${ }^{1}$

16 Shushanhu road, Hefei, Anhui, 230031, China

17 Email address: zhx@hmfl.ac.cn

18 Junfeng Wang $1,2,5$

19 Shushanhu road, Hefei, Anhui, 230031, China

20 Email address: junfeng@hmfl.ac.cn

21

\section{Abstract}

Tyrosine autophosphorylation plays a crucial regulatory role in the kinase activities of fibroblast growth factor receptors (FGFRs), and in the recruitment and activation of downstream intracellular signaling pathways. Biophysical and biochemical investigations of FGFR kinase domains in membrane environments offer key insights into phosphorylation mechanisms. Hence, we constructed nickel chelating nanodiscs based on a 22-residue peptide. The spontaneous anchoring of N-terminal $\mathrm{His}_{6}$-tagged FGFR1c kinase domain (FGFR1K) onto peptide nanodiscs grants FGFR1K orientations occurring on native plasma membranes. Following membrane incorporation, the autophosphorylation of FGFR1K, as exemplified by Y653 and Y654 in the Aloop and the total tyrosine phosphorylation, increase significantly. This in vitro reconstitution system may be applicable to studies of other membrane associated phenomena. 
40 Introduction

41 As members of the receptor tyrosine kinases (RTKs) family, fibroblast growth factor receptors

42 (FGFRs) play important roles as regulators of proliferation, differentiation, migration, and survival

43 in various cell types (Eswarakumar et al. 2005; Mikhaylenko et al. 2018). Accordingly,

44 deregulation of FGFR signaling contributes to various pathological conditions and developmental

45 syndromes (Tiong et al. 2013; Turner \& Grose 2010). FGF ligands bind the extracellular domain

46 of FGFR with facilitating cofactors, such as heparin sulfate and klothos (Goetz et al. 2012;

47 Schlessinger et al. 2000), and induce receptor homo-dimerization and subsequent activation of

48 cytoplasmic tyrosine kinase domains by tyrosine autophosphorylation at multiple sites. At least

49 seven tyrosine autophosphorylation sites have been described in FGFR1 (Y463, Y583, Y585,

50 Y653, Y654, Y730 and Y766; Fig. 1A) (Mohammadi et al. 1996). Phosphorylation of the A-loop

51 residues Y653 and Y654 (highlighted in Fig. 1B) (Bae et al. 2010; Bae et al. 2009) is critical for

52 upregulation of kinase activity (Furdui et al. 2006). Moreover, phosphorylation sites of tyrosine

53 residues in other parts of the cytoplasmic region serve as specific binding sites for downstream

54 signaling molecules containing Src Homology 2 (SH2) or phospho-tyrosine binding domains,

55 resulting in the activation of specific signaling pathways (Babina \& Turner 2017; Bae et al. 2009).

56 To investigate the mechanisms of RTK autophosphorylation, many biological and biophysical

57 studies have been performed using analyses of isolated kinase domains in aqueous solution (Klein

58 et al. 2015; Kobashigawa et al. 2015). Yet other studies of RTKs show that isolated kinase domains

59 fail to reproduce in vivo observations (Bae et al. 2010). This largely reflects the importance of cell

60 membrane localization of kinase domains, which is essential for promoting receptor dimerization

61 and cooperative cross-receptor autophosphorylation.

62 Given the importance of protein-membrane interactions, accurate experimental mimicry of 63 membrane anchors is necessary for studies of RTK kinase domains. A strategy for binding poly- 
64 histidine-tagged proteins to small unilamellar vesicles or liposomes was previously developed

65 using nickel chelating lipids (Esposito et al. 2008; Zhang et al. 2006). Weis and coworkers

66 pioneered the use of this strategy in their studies of a prokaryotic signal transduction system that

67 mediates bacterial chemotaxis (Montefusco et al. 2007; Shrout et al. 2003). In addition, Monsey

68 and coworkers used nickel chelating liposomes to form heterodimers of epidermal growth factor

69 receptors (EGFR) between Her4 and Her2/neu tyrosine kinase domains mimicking the in vivo

70 behaviors of full-length receptors following ligand binding. Their study showed that addition of

71 nickel chelating liposomes to the Her4 kinase domain resulted in a 40-fold increase in kinase

72 activity (Monsey et al. 2010).

73 Nanodiscs are discoidal nanomembrane particles with a planar phospholipid bilayer enwrapped

74 by proteins such as apolipoprotein A-I or membrane scaffolding proteins (MSP) (Parmar et al.

75 2016). Nanodiscs have been widely used for analyzing structures and functions of membrane

76 proteins by dispersing them in solution (Hagn et al. 2018; Nasr et al. 2017; Rouck et al. 2017). In

77 addition, the lipid compositions of nanodiscs can be controlled precisely providing a nanoscale

78 membrane surface for investigating various membrane recognition events (Denisov \& Sligar

79 2017). To simplify the preparation procedure at the same time maintaining the stabilization

80 provided by nanodiscs, a new strategy, named peptide nanodisc, has been developed. This is based

81 on the use of synthetic peptides mimicking the amphipathic helices of Apolipoprotein I, whereas

82 the nanodisc sizes can be conveniently controlled by varying the lipid/ peptide ratio (Imura et al.

83 2014; Miyazaki et al. 2010).

84 Using a 22 amino-acid peptide derived from Apolipoprotein I protein (Zhang et al. 2016),

85 herein, we introduced nickel chelating lipids into peptide nanodisc to generate a nanoscale 2D-

86 membrane mimics for studying FGFR1 kinase domain (FGFR1K) activity. Our data show that 
87 histidine-tagged FGFR1K can be conveniently assembled onto this peptide-based nickel chelating

88 nanodiscs. The autophosphorylation of tyrosines, especially Y653 and Y654 on the activated loop

89 of kinase region, will be studied using nanoscale membrane templates.

90 Materials \& Methods

91 Chemicals and Reagents

92 1, 2-dimyristoyl-sn-glycero-3-phosphocholine (DMPC) and 1, 2-dioleoyl-sn-glycero-3-[(N-(593 amino-1-carboxypentyl) iminodiacetic acid) succinyl] (nickel salt) (DGS-NTA(Ni)) were 94 purchased from Avanti Polar Lipids. Antibodies against FGF receptor 1 (D8E4; cat. 9740), FGFR 95 phospho-Tyr-653/4 (cat. 3476), and phospho-tyrosine (P-Tyr-1000; cat. 8954) were purchased 96 from Cell Signaling Technology, Inc. Antibodies against FGFR1 phospho-Tyr-653 (cat. 97 ab173305) and phospho-Tyr-654 (cat. ab59194) were purchased from Abcam. Dithiobis 98 (succinimidyl propionate) (DSP; cat. C110213) was purchased from Sangon Biotech. The 2299 residue peptide PVLDLFRELLNELLEALKQKLK was synthesized by GL Biochem (Shanghai) 100 Ltd.

101 FGFR1K Expression and Purification

102 DNA encoding the FGFR1c kinase domain (residues 458-765; FGFR1K) was cloned into the 103 pFastBacl vector (Invitrogen) using BamHI and HindIII restriction sites. All constructs contained 104 an N-terminal $\mathrm{His}_{6}$ tag, and were verified in DNA sequencing analyses. Recombinant bacmids 105 were then transfected into Sf9 cells using the Bac-to-Bac expression system (Zhang et al. 2018). 106 Briefly, Sf9 cells were infected with recombinant baculovirus and expressed the recombinant 107 protein. Transfected cells were harvested after 2 days by centrifugation at $3000 \mathrm{~g}$ and were then 108 lysed in buffer A containing $20 \mathrm{mM}$ Tris, $\mathrm{pH}$ 8.0, $400 \mathrm{mM} \mathrm{NaCl}, 10 \%$ glycerol, protease inhibitor 109 cocktail (Roche), $10 \mathrm{ng} / \mathrm{ml} \mathrm{PMSF}, 100 \mu \mathrm{M} \mathrm{Na}_{3} \mathrm{VO}_{4}$, and 1\% triton X-100 on ice for $30 \mathrm{~min}$. After 110 centrifugation at $50000 \mathrm{rpm}$ for $45 \mathrm{~min}$, proteins were purified using a Ni-NTA column and size 
111 exclusion chromatography with a Superdex 200 GL 16/60 column (GE Healthcare) at a flow rate

112 of $1 \mathrm{ml} / \mathrm{min}$ at $4^{\circ} \mathrm{C} .20 \mathrm{mM}$ Tris, $\mathrm{pH} 8.0,400 \mathrm{mM} \mathrm{NaCl}$ was used as the running buffer. Target

113 fractions were pooled and concentrated, finally stored at $-80^{\circ} \mathrm{C}$.

114 Preparation of NTA(Ni)-nanodiscs

115 To prepare nanodiscs, 95\% DMPC and 5\% DGS-NTA(Ni) powders were dissolved in buffer 116 containing $10 \mathrm{mM}$ potassium phosphate $(\mathrm{pH} 7.4)$ to prepare $20 \mathrm{mg} / \mathrm{mL}$ lipid stock solution. 117 Subsequently, 22-amino acid peptides were dissolved in buffer containing $40 \mathrm{mM}$ potassium 118 phosphate, ( $\mathrm{pH} 7.4)$, to make a $10 \mathrm{mg} / \mathrm{mL}$ peptide stock solutions. Peptide and lipids were then 119 mixed at indicated ratios and incubated at $50^{\circ} \mathrm{C}$ for $10 \mathrm{~min}$ and then at room temperature for 10 120 min. This procedure was repeated 3 to 5 times until the solution became clear, and three freeze and 121 thaw cycles were then performed between $-80^{\circ} \mathrm{C}$ and room temperature to homogenize the 122 nanodiscs (Zhang et al. 2016). The resulting nanodisc solutions were then characterized using size 123 exclusion chromatography (SEC) with a Superdex 200 GL 10/300 column (GE Healthcare) at a 124 flow rate of $0.5 \mathrm{ml} / \mathrm{min}$ at room temperature. $20 \mathrm{mM} \mathrm{PB},(\mathrm{pH} 7.4), 50 \mathrm{mM} \mathrm{NaCl}$ was used as the running buffer. Target fractions were pooled and concentrated, prepared for kinase assays.

\section{Transmission Electron Microscopy (TEM)}

127 The specimens for transmission electron microscopy (TEM) was prepared by negative-stain 128 technique. Carbon-coated copper grids (200 mesh) were cleaned for $5 \mathrm{~min}$ in plasma cleaner. 129 Nanodisc samples were incubated on the grids for $1 \mathrm{~min}$, and the excess was removed by blotting 130 with filter paper. The grids were then stained with phosphorotungstic acid for $20 \mathrm{~s}$ and excess 131 solution was removed by blotting. Air-dried samples were stored in a desiccator until observation. 132 All TEM measurements were carried out using a FEI Tecnai Spirit tandem electron microscope 133 (TEM; $100 \mathrm{kV})$. The particle diameter distribution and discoidal shape analysis were based on the 
134 2D class averaging using EMAN2. For each peptide nanodiscs, at least 3000 particles were 135 selected and classified.

136 Kinase Assays

137 The activities of the kinase domains that were attached to nanodiscs were assayed by pre138 incubating FGFR1K with nanodiscs of various diameters at $4^{\circ} \mathrm{C}$ overnight. A Superdex 200 10/300 139 GL column was used to separate FGFR1K NTA(Ni)-nanodiscs from FGFR1K and empty 140 nanodiscs prior to the start of kinase assays. Control samples of the kinase domain in solution 141 without nanodiscs were also assayed using the same protocols. Kinase activity assays were then 142 performed with $1.5 \mathrm{nM}$ purified FGFR1K or FGFR1K NTA(Ni)-nanodiscs in buffer containing 5 $143 \mathrm{mM}$ ATP, $10 \mathrm{mM} \mathrm{MgCl} 2$ in $20 \mathrm{mM}$ Tris and $200 \mathrm{mM} \mathrm{NaCl}(\mathrm{pH} 8.0)$ at room temperature.

144 Reactions were allowed to proceed at room temperature for indicated times and were then 145 quenched using $50 \mathrm{mM}$ EDTA. Subsequently, $6 \times$ loading buffer was added to each sample and 146 proteins were loaded onto polyacrylamide-sodium dodecyl sulfate gels. After electrophoretic 147 separation, proteins were transferred to nitrocellulose membranes, and these were then blocked 148 using 5\% BSA in TBS-T. Proteins were probed with various antibodies and the resulting blots 149 were visualized using chemiluminescence autoradiography. The results were quantitated by 150 ImageJ analysis. Data represent the average of at least four experiments. Statistical tests were 151 conducted using either unpaired two-tailed Student's t test or one-way analysis of variance with 152 post-hoc Dunnett's test. Error bars depict the S.D. P values of *p $<0.05,{ }^{* *} \mathrm{p}<0.01$, and ${ }^{* * *} \mathrm{p}<$ 1530.001 were considered to be statistically significant.

\section{Isothermal Titration Calorimetry (ITC)}

155 Isothermal titration calorimetry measurements were performed on a MicroCalITC200 instrument 156 (MicroCal GE, USA). FGFR1K proteins were diluted into $0.04 \mathrm{mM}$ with the buffer of $20 \mathrm{mM} \mathrm{PB}$, 
$157 \mathrm{pH} 7.4,50 \mathrm{mM} \mathrm{NaCl}$ then titrated into the $\mathrm{NTA}(\mathrm{Ni})$-Nanodiscs solution with the same buffer at

$15825^{\circ} \mathrm{C}$. All buffers and samples were centrifuged at $13000 \mathrm{rpm}$ for $30 \mathrm{~min}$ to minimize possible

159 bubble formation during ITC stirrings. A control titration, consisting of the same titration buffer

160 solution in the sample cell, was subtracted from each experimental titration to account for heat of

161 dilution. The corrected binding isotherms were fitted to obtain the binding constant (K), the

162 number of binding sites $(\mathrm{N})$, enthalpy change $(\Delta \mathrm{H})$ and entropy change $(\Delta \mathrm{S})$, by using Malvern

163 MicroCal PEAQ-ITC software.

\section{Results}

\section{Assembly of Nanodises of Various Diameters}

166 Membrane mimic systems are prerequisites for studies of the in vitro effects of membrane binding 167 on FGFR1K. Herein, we assembled nanodiscs using the amphipathic 22-residue peptide 168 PVLDLFRELLNELLEALKQKLK (22A) based on an amphipathic $\alpha$-helical segment of the apolipoprotein A-I (Apo A-I) (Zhang et al. 2016). In contrast with the commonly used membrane

170 scaffold protein (MSP) from Apo A-I, which forms fix-sized nanodiscs, 22A allows assembly of 171 size controllable nanodiscs using a comparatively simple procedure. We prepared nanodiscs of 172 three different sizes by varying molar ratios of lipid (DMPC) to peptide. At molar ratios of 1:9, 173 1:3, and 1:1, single peaks were present on SEC elution profiles at 14.1, 12.6, and $10.9 \mathrm{ml}$, each 174 indicating single assembled forms with homogeneous size distributions (Fig. 2A). The elution peak 175 position of peptide nanodisc of 1:3 was similar to that of the generally used MSP $\Delta \mathrm{H} 5$ nanodiscs, 176 indicating similar nanodisc sizes (Supplementary Fig. S1). TEM of 22A nanodiscs (Fig. 2B-D) 177 provide a direct evidence that peptide nanodiscs form disc-like assembly. The appearance of 178 nanodisc stacking at molar ratio of 1:1 (Supplementary Fig. S2) was an artifact associated with 179 negative staining sample treatment, which were also reported in other study( $\mathrm{R}$ et al. 2017). The 
180 average diameters from 2D class average analysis are $6.3 \pm 1.1 \mathrm{~nm}, 8.6 \pm 1.2 \mathrm{~nm}$, and $12.0 \pm 1.8 \mathrm{~nm}$, 181 respectively. DLS analyses (Supplementary Fig. S3) also verified that the diameters of assembled

182 nanodiscs change linearly with lipid/peptide molar ratios, although the sizes derived are slightly 183 smaller than those from TEM (Supplementary Table S1). Such a discrepancy in sizes is likely 184 caused by differences in sample treatment as well as data analysis.

185 Chelating FGFR1K onto NTA(Ni)-nanodiscs

186 To attach the kinase to NTA(Ni)-nanodiscs and determine in vitro activity, we generated a 187 FGFR1K (residues 458-765) construct with an $\mathrm{N}$-terminal $\mathrm{His}_{6}$ tag. The protein was expressed in 188 a Sf9 cell expression system and was purified using Ni affinity chromatography followed by size 189 exclusion chromatography (Supplementary Fig. S4). Nanodiscs were then assembled using neutral 190 DMPC lipid containing 5\% NTA-Ni-DGS. His ${ }_{6}$ tagged FGFR1K was incubated with various 191 NTA(Ni)-nanodiscs overnight and the resulting complexes were collected using SEC. The purified 192 FGFR1K possessed enzyme activity, and the phosphorylation happened at tyrosine sites can be 193 monitored using specific antibodies (Supplementary Fig. S6). The His-tagged FGFR1K can bind 194 to NTA(Ni)-nanodiscs (1:1) as indicated in the SEC profile of Fig. 3A, where a higher molecular 195 weight complex forms and therefore elutes faster (red line with an elution peak at $10.2 \mathrm{ml}$ ) than 196 NTA(Ni)-nanodiscs (1:1) only (black line with an elution peak at $10.9 \mathrm{ml}$ ) sample and FGFR1K 197 only sample (blue line with an elution peak at $15.1 \mathrm{ml}$ ). The co-presence of FGFR1K and 22A 198 peptide bands in SDS PAGE confirms the binding of FGFR1K to NTA(Ni)-nanodiscs. FGFR1K 199 and peptide NTA(Ni)-nanodisc forms stable complex with a dissociation constant of $2.4 \mu \mathrm{M}$ as 200 determined by the isothermal titration calorimetry (ITC) experiments (Fig. 3B). The effects of 201 membrane binding were characterized by monitoring total tyrosine phosphorylation and that of 202 Y653/4 in the A-loop of FGFR1K (Fig. 3C). The total tyrosine phosphorylation and the 
203 phosphorylations of $\mathrm{Y} 653 / 4$ in the presence of NTA(Ni)-nanodiscs of 1:3 and 1:9 are less or 204 comparable to the aqueous control. Among the three peptide nanodiscs assembled, the highest 205 autophosphorylation activity of FGFR1K was achieved with nanodisc of 1:1. According to the 206 cross-linking results, the highest FGFR1K oligomer to monomer ratio (Supplementary Fig. S7) 207 also appears in the presence of nanodisc (1:1), suggesting a correlation between FGFR1K 208 autophosphorylation activity and nannodisc sizes.

\section{Kinetic Analysis of FGFR1K Autophosphorylation on NTA(Ni)-nanodises}

210 To characterize the autophosphorylation kinetics, we monitored phosphorylation over time using 211 anti-p-tyrosine antibodies (Supplementary Fig. S8). As shown in Fig. 4A, the total phosphorylation 212 plateaued at $1 \mathrm{~h}$, and membrane binding significantly enhanced ( 2 folds) tyrosine 213 autophosphorylation. The phosphorylation of the A-loop residues Y653 and Y654, which has been 214 reported to be critical for upregulation of kinase activity (Turner \& Grose 2010), were also 215 monitored by western blotting analyses using antibodies against p-Y653 and p-Y654. In aqueous 216 solution, minor changes were observed for Y653 phosphorylation levels over time, whereas the 217 presence of nanodiscs led to a maximal 10-fold increase in phosphorylation of Y653 over $2 \mathrm{~h}$, 218 which is 5 -fold greater than that in aqueous controls (Fig. 4B). It takes only a few minutes for 219 Y654 to reach a phosphorylation plateau in aqueous solution. In the presence of peptide nanodiscs, 220 the phosphorylation of Y654 continues to grow, and the phosphorylation at $2 \mathrm{~h}$ is about 4-fold 221 compared with the aqueous control (Fig. 4C). The Y654 phosphorylation increases 17 times 222 comparing to its initial phosphorylation level. The enhancements of both Y653 and Y654 223 phosphorylation strongly indicate that, the binding of FGFR1K on 2D membrane nanodiscs 224 facilitates Y653 and Y654 activation.

\section{Discussion}


226 Tyrosine autophosphorylation of RTKs plays a critical role in the regulation of kinase activities 227 and in the recruitment and activation of downstream substrates. Many studies of kinase-only 228 domains in aqueous environments, nevertheless, missed the conditions provided by native 229 membrane surface that is essential for autophosphorylation. For example, autophosphorylation 230 requires the kinase domains to orient and cooperate respectively on dimer interfaces, differing 231 considerably from the random collisions that occur in aqueous environments. However, the 232 hydrophobicity associated with the trans-membrane fragment of full-length FGFR hampers protein 233 expression and purification, and subsequent biophysical and biochemical analyses. These 234 conditions necessitate mimicry of cell membrane environments to promote receptor dimerization 235 and cooperative cross-receptor interactions.

236 Nanodiscs provide nanoscale surfaces on which functional complexes can be assembled with 237 lipid membranes, offering a good membrane mimic system for investigating membrane-mediated 238 molecular recognition events. In contrast with fixed-sized nanodiscs that are assembled with MSP, the present 22A-peptide based nanodisc assembly allows easy and accurate adjustments of nanodisc sizes by changing lipid/peptide ratios. Further addition of nickel chelating lipid allowed 241 the anchoring of N-terminal His 6 tagged FGFR1K onto lipid membrane even in the absence of 242 FGFR transmembrane domain. These protein-nanodisc assemblies led to relatively well-defined

243 samples that are amenable to various analyses, including DLS, TEM, and SEC. This approach also 244 has promise in other systems involving membrane associated proteins.

245 FGFRs transduce biochemical signals via lateral dimerization and autophosphorylation on 246 plasma membranes. To this end, the kinase domain of one FGFR molecule serves as an active 247 enzyme, and the other, its substrate. In aqueous solution, although kinase domains diffuse freely 248 and conveniently cross-linked (Supplementary Fig. S7), the phosphorylation level is rather low 
249 (Fig. 3C), and occurs only when two kinase domains diffuse and collide with appropriate 250 orientation relative to each other. The presence of NTA(Ni)-nanodiscs, on the other hand, restricts 251 kinase domains to diffuse in a 2D space, and increases the chances of kinase collision and reaction. 252 Moreover, the membrane anchoring also helps in coordinating the kinase enzyme and kinase 253 substrate, into the appropriate orientations for phosphorylation. From our data, only NTA(Ni)254 nanodiscs at a lipid:peptide ratio of 1:1 exhibited significant enhancements of kinase 255 oligomerization and activation, indicating the necessary of sufficient membrane surface for 256 autophosphorylation. The flexibility and convenience of peptide nanodiscs in tuning membrane 257 areas, therefore make it a versatile membrane mimic system in studying various membrane 258 associated phenomena.

259 Autophosphorylation of the tyrosine residues of FGFR1K is a precisely ordered process and is 260 kinetically driven, as shown in mass spectroscopy analyses (Lew et al. 2009). These studies 261 suggest that each step of the process is accompanied by a different orientation of catalytic kinase 262 relative to substrate kinase. FGFR phosphorylation kinetics on the membrane is likely more 263 complicated, warranting further studies to determine the order of phosphorylation reactions on 264 membranes, and how membranes affect phosphorylation kinetics. Although the present western 265 blotting analyses of the kinetics of autophosphorylation at tyrosine sites on nanodiscs are semi 266 quantitative, our nanodiscs significantly increased phosphorylation levels of all tyrosine residues 267 and those at Y653 and Y654. Moreover, in the presence of nanodiscs, Y653 phosphorylation 268 plateaued after $1 \mathrm{~h}$, whereas the Y654 phosphorylation continue to increase (Fig. 4), indicating 269 phosphorylation of Y653 happens earlier than that of Y654. This is consistent with previous report 270 that Y653 is the first site to be phosphorylated and stimulates kinase activity by 50- to 100 -fold 271 (Lew et al. 2009). However, due to the complexity of the autophosphorylation mechanism and the 
272 lack of available antibodies for specific phosphor-tyrosine forms, we were not able to determine

273 the order of autophosphorylation precisely. The regulatory effects of membranes on

274 autophosphorylation at various tyrosine sites may be better characterized using techniques such as

275 mass spectroscopy analysis.

276 Conclusions

277 In conclusion, we developed an NTA(Ni)-nanodiscs system for which the nanodisc size can be

278 finely adjusted. An N-terminal His $_{6}$-tagged FGFR1K anchored spontaneously onto the membrane

279 with orientations that mimic those in plasma membranes. The 2D membrane surfaces provided by

280 peptide nanodiscs significantly enhanced the phosphorylation rates of Y653 and Y654 in FGFR1K

281 A-loop. The present system has potential applications to in vitro studies of other membrane

282 associated phenomena.

283

284

285

286

287

288

289

290

291

292

293

294

295

296

297

298

299

300

301

302

303

304

305

306

307

308

309

\section{References}

Babina IS, and Turner NC. 2017. Advances and challenges in targeting FGFR signalling in cancer. Nature Reviews Cancer 17:318-332. 10.1038/nrc.2017.8

Bae JH, Boggon TJ, Tome F, Mandiyan V, Lax I, and Schlessinger J. 2010. Asymmetric receptor contact is required for tyrosine autophosphorylation of fibroblast growth factor receptor in living cells. Proceedings of the National Academy of Sciences of the United States of America 107:2866-2871. 10.1073/pnas.0914157107

Bae JH, Lew ED, Yuzawa S, Tome F, Lax I, and Schlessinger J. 2009. The Selectivity of Receptor Tyrosine Kinase Signaling Is Controlled by a Secondary SH2 Domain Binding Site. Cell 138:514-524. 10.1016/j.cell.2009.05.028

Denisov IG, and Sligar SG. 2017. Nanodiscs in Membrane Biochemistry and Biophysics. Chem Rev 117:4669-4713. 10.1021/acs.chemrev.6b00690

Esposito EA, Shrout AL, and Weis RM. 2008. Template-directed self-assembly enhances RTK catalytic domain function. Journal of Biomolecular Screening 13:810-816. 10.1177/1087057108322062

Eswarakumar VP, Lax I, and Schlessinger J. 2005. Cellular signaling by fibroblast growth factor receptors. Cytokine \& Growth Factor Reviews 16:139-149. 10.1016/j.cytogfr.2005.01.001

Furdui CM, Lew ED, Schlessinger J, and Anderson KS. 2006. Autophosphorylation of FGFR1 kinase is mediated by a sequential and precisely ordered reaction. Molecular Cell 21:711-717. 10.1016/j.molcel.2006.01.022

Goetz R, Ohnishi M, Kir S, Kurosu H, Wang L, Pastor J, Ma JH, Gai WM, Kuro-o M, Razzaque MS, and Mohammadi M. 2012. Conversion of a Paracrine Fibroblast Growth Factor into an Endocrine Fibroblast Growth Factor. Journal of Biological Chemistry 287:29134-29146. 10.1074/jbc.M112.342980

Imura T, Tsukui Y, Taira T, Aburai K, Sakai K, Sakai H, Abe M, and Kitamoto D. 2014. Surfactant-like properties of an amphiphilic alpha-helical peptide leading to lipid nanodisc formation. Langmuir 30:4752-4759. 10.1021/la500267b 
310

311

312

313

314

315

316

317

318

319

320

321

322

323

324

325

326

327

328

329

330

331

332

333

334

335

336

337

338

339

340

341

342

343

344

345

346

347

348

349

350

351

352

353

354

355

356

357

358

359

Klein T, Vajpai N, Phillips JJ, Davies G, Holdgate GA, Phillips C, Tucker JA, Norman RA, Scott AD, Higazi DR, Lowe D, Thompson GS, and Breeze AL. 2015. Structural and dynamic insights into the energetics of activation loop rearrangement in FGFR1 kinase. Nature Communications 6. ARTN 7877

10.1038/ncomms8877

Kobashigawa Y, Amano S, Yokogawa M, Kumeta H, Morioka H, Inouye M, Schlessinger J, and Inagaki F. 2015. Structural analysis of the mechanism of phosphorylation of a critical autoregulatory tyrosine residue in FGFR1 kinase domain. Genes to Cells 20:860-870. 10.1111/gtc.12277

Lew ED, Furdui CM, Anderson KS, and Schlessinger J. 2009. The Precise Sequence of FGF Receptor Autophosphorylation Is Kinetically Driven and Is Disrupted by Oncogenic Mutations. Science Signaling 2. ARTN ra6

10.1126/scisignal.2000021

Mikhaylenko DS, Alekseev BY, Zaletaev DV, Goncharova RI, and Nemtsova MV. 2018. Structural Alterations in Human Fibroblast Growth Factor Receptors in Carcinogenesis. BiochemistryMoscow 83:930-943. 10.1134/S0006297918080059

Miyazaki M, Tajima Y, Handa T, and Nakano M. 2010. Static and dynamic characterization of nanodiscs with apolipoprotein A-I and its model peptide. $J$ Phys Chem B 114:12376-12382. 10.1021/jp102074b

Mohammadi M, Dikic I, Sorokin A, Burgess WH, Jaye M, and Schlessinger J. 1996. Identification of six novel autophosphorylation sites on fibroblast growth factor receptor 1 and elucidation of their importance in receptor activation and signal transduction. Molecular and Cellular Biology 16:977989.

Monsey J, Shen W, Schlesinger PS, and Bose R. 2010. Her4 and Her2/neu tyrosine kinase domains dimerize and activate in a reconstituted in vitro system. Cancer Research 70. 10.1158/1538-7445.Am103122

Montefusco DJ, Shrout AL, Besschetnova TY, and Weis RM. 2007. Formation and activity of templateassembled receptor signaling complexes. Langmuir 23:3280-3289. 10.1021/1a062717r

Parmar MJ, Lousa Cde M, Muench SP, Goldman A, and Postis VL. 2016. Artificial membranes for membrane protein purification, functionality and structure studies. Biochem Soc Trans 44:877-882. 10.1042/BST20160054

Rouck JE, Krapf JE, Roy J, Huff HC, and Das A. 2017. Recent advances in nanodisc technology for membrane protein studies (2012-2017). FEBS Lett 591:2057-2088. 10.1002/1873-3468.12706

Schlessinger J, Plotnikov AN, Ibrahimi OA, Eliseenkova AV, Yeh BK, Yayon A, Linhardt RJ, and Mohammadi M. 2000. Crystal structure of a ternary FGF-FGFR-heparin complex reveals a dual role for heparin in FGFR binding and dimerization. Molecular Cell 6:743-750.

Shrout AL, Montefusco DJ, and Weis RM. 2003. Template-directed assembly of receptor signaling complexes. Biochemistry 42:13379-13385. 10.1021/bi0352769

Tiong KH, Mah LY, and Leong CO. 2013. Functional roles of fibroblast growth factor receptors (FGFRs) signaling in human cancers. Apoptosis 18:1447-1468. 10.1007/s10495-013-0886-7

Turner N, and Grose R. 2010. Fibroblast growth factor signalling: from development to cancer. Nature Reviews Cancer 10:116-129. 10.1038/nrc2780

Zhang M, Huang R, Ackermann R, Im SC, Waskell L, Schwendeman A, and Ramamoorthy A. 2016. Reconstitution of the Cytb5-CytP450 Complex in Nanodiscs for Structural Studies using NMR Spectroscopy. Angew Chem Int Ed Engl 55:4497-4499. 10.1002/anie.201600073

Zhang S, Huang YH, Zhu JL, Shan LY, Gao JF, Zhang YH, Yu N, Yang L, and Huang J. 2018. Expression of hNeuritin protein in a baculovirus expression system and the analysis of its activity. Gene 647:129-135. 10.1016/j.gene.2018.01.026

Zhang XW, Gureasko J, Shen K, Cole PA, and Kuriyan J. 2006. An allosteric mechanism for activation of the kinase domain of epidermal growth factor receptor. Cell 125:1137-1149.

10.1016/j.cell.2006.05.013

Peer] reviewing PDF | (2019:01:34565:1:1:NEW 30 May 2019) 
360

361

362

363

364

365

366

367

368

369

370

371

372

373

374

375

376

377

378

379

380

381

382

383

384

385

386

387

388

389

390

391

392

393

394

395

396

397

398

399

400

401

402

403

404

405

406

407

408

409

410

Babina IS, and Turner NC. 2017. Advances and challenges in targeting FGFR signalling in cancer. Nature Reviews Cancer 17:318-332. 10.1038/nrc.2017.8

Bae JH, Boggon TJ, Tome F, Mandiyan V, Lax I, and Schlessinger J. 2010. Asymmetric receptor contact is required for tyrosine autophosphorylation of fibroblast growth factor receptor in living cells. Proceedings of the National Academy of Sciences of the United States of America 107:2866-2871. 10.1073/pnas.0914157107

Bae JH, Lew ED, Yuzawa S, Tome F, Lax I, and Schlessinger J. 2009. The Selectivity of Receptor Tyrosine Kinase Signaling Is Controlled by a Secondary SH2 Domain Binding Site. Cell 138:514-524. 10.1016/j.cell.2009.05.028

Denisov IG, and Sligar SG. 2017. Nanodiscs in Membrane Biochemistry and Biophysics. Chem Rev 117:4669-4713. 10.1021/acs.chemrev.6b00690

Esposito EA, Shrout AL, and Weis RM. 2008. Template-directed self-assembly enhances RTK catalytic domain function. Journal of Biomolecular Screening 13:810-816. 10.1177/1087057108322062

Eswarakumar VP, Lax I, and Schlessinger J. 2005. Cellular signaling by fibroblast growth factor receptors. Cytokine \& Growth Factor Reviews 16:139-149. 10.1016/j.cytogfr.2005.01.001

Furdui CM, Lew ED, Schlessinger J, and Anderson KS. 2006. Autophosphorylation of FGFR1 kinase is mediated by a sequential and precisely ordered reaction. Molecular Cell 21:711717. 10.1016/j.molcel.2006.01.022

Goetz R, Ohnishi M, Kir S, Kurosu H, Wang L, Pastor J, Ma JH, Gai WM, Kuro-o M, Razzaque MS, and Mohammadi M. 2012. Conversion of a Paracrine Fibroblast Growth Factor into an Endocrine Fibroblast Growth Factor. Journal of Biological Chemistry 287:29134-29146. 10.1074/jbc.M112.342980

Hagn F, Nasr ML, and Wagner G. 2018. Assembly of phospholipid nanodiscs of controlled size for structural studies of membrane proteins by NMR. Nat Protoc 13:79-98. 10.1038/nprot.2017.094

Imura T, Tsukui Y, Taira T, Aburai K, Sakai K, Sakai H, Abe M, and Kitamoto D. 2014. Surfactantlike properties of an amphiphilic alpha-helical peptide leading to lipid nanodisc formation. Langmuir 30:4752-4759. 10.1021/la500267b

Klein T, Vajpai N, Phillips JJ, Davies G, Holdgate GA, Phillips C, Tucker JA, Norman RA, Scott AD, Higazi DR, Lowe D, Thompson GS, and Breeze AL. 2015. Structural and dynamic insights into the energetics of activation loop rearrangement in FGFR1 kinase. Nature Communications 6. ARTN 7877

10.1038/ncomms8877

Kobashigawa Y, Amano S, Yokogawa M, Kumeta H, Morioka H, Inouye M, Schlessinger J, and Inagaki F. 2015. Structural analysis of the mechanism of phosphorylation of a critical autoregulatory tyrosine residue in FGFR1 kinase domain. Genes to Cells 20:860-870. 10.1111/gtc. 12277

Lew ED, Furdui CM, Anderson KS, and Schlessinger J. 2009. The Precise Sequence of FGF Receptor Autophosphorylation Is Kinetically Driven and Is Disrupted by Oncogenic Mutations. Science Signaling 2. ARTN ra6

10.1126/scisignal.2000021

Mikhaylenko DS, Alekseev BY, Zaletaev DV, Goncharova RI, and Nemtsova MV. 2018. Structural Alterations in Human Fibroblast Growth Factor Receptors in Carcinogenesis. Biochemistry-Moscow 83:930-943. 10.1134/S0006297918080059

Miyazaki M, Tajima Y, Handa T, and Nakano M. 2010. Static and dynamic characterization of nanodiscs with apolipoprotein A-I and its model peptide. J Phys Chem B 114:1237612382. 10.1021/jp102074b

Mohammadi M, Dikic I, Sorokin A, Burgess WH, Jaye M, and Schlessinger J. 1996. Identification of six novel autophosphorylation sites on fibroblast growth factor receptor 1 and

Peer) reviewing PDF | (2019:01:34565:1:1:NEW 30 May 2019) 
411

412

413

414

415

416

417

418

419

420

421

422

423

424

425

426

427

428

429

430

431

432

433

434

435

436

437

438

439

440

441

442

443

444

445

446

447

448

449

450 elucidation of their importance in receptor activation and signal transduction. Molecular and Cellular Biology 16:977-989.

Monsey J, Shen W, Schlesinger PS, and Bose R. 2010. Her4 and Her2/neu tyrosine kinase domains dimerize and activate in a reconstituted in vitro system. Cancer Research 70. 10.1158/1538-7445.Am10-3122

Montefusco DJ, Shrout AL, Besschetnova TY, and Weis RM. 2007. Formation and activity of template-assembled receptor signaling complexes. Langmuir 23:3280-3289. 10.1021/la062717r

Nasr ML, Baptista D, Strauss M, Sun ZJ, Grigoriu S, Huser S, Pluckthun A, Hagn F, Walz T, Hogle JM, and Wagner G. 2017. Covalently circularized nanodiscs for studying membrane proteins and viral entry. Nat Methods 14:49-52. 10.1038/nmeth.4079

Parmar MJ, Lousa Cde M, Muench SP, Goldman A, and Postis VL. 2016. Artificial membranes for membrane protein purification, functionality and structure studies. Biochem Soc Trans 44:877-882. 10.1042/BST20160054

R BK, Zhu L, Hebert H, and Jegerschold C. 2017. Method to Visualize and Analyze Membrane Interacting Proteins by Transmission Electron Microscopy. J Vis Exp. 10.3791/55148

Rouck JE, Krapf JE, Roy J, Huff HC, and Das A. 2017. Recent advances in nanodisc technology for membrane protein studies (2012-2017). FEBS Lett 591:2057-2088. 10.1002/18733468.12706

Schlessinger J, Plotnikov AN, Ibrahimi OA, Eliseenkova AV, Yeh BK, Yayon A, Linhardt RJ, and Mohammadi M. 2000. Crystal structure of a ternary FGF-FGFR-heparin complex reveals a dual role for heparin in FGFR binding and dimerization. Molecular Cell 6:743-750.

Shrout AL, Montefusco DJ, and Weis RM. 2003. Template-directed assembly of receptor signaling complexes. Biochemistry 42:13379-13385. 10.1021/bi0352769

Tiong $\mathrm{KH}$, Mah LY, and Leong CO. 2013. Functional roles of fibroblast growth factor receptors (FGFRs) signaling in human cancers. Apoptosis 18:1447-1468. 10.1007/s10495-0130886-7

Turner N, and Grose R. 2010. Fibroblast growth factor signalling: from development to cancer. Nature Reviews Cancer 10:116-129. 10.1038/nrc2780

Zhang M, Huang R, Ackermann R, Im SC, Waskell L, Schwendeman A, and Ramamoorthy A. 2016. Reconstitution of the Cytb5-CytP450 Complex in Nanodiscs for Structural Studies using NMR Spectroscopy. Angew Chem Int Ed Engl 55:4497-4499. 10.1002/anie.201600073

Zhang S, Huang YH, Zhu JL, Shan LY, Gao JF, Zhang YH, Yu N, Yang L, and Huang J. 2018. Expression of hNeuritin protein in a baculovirus expression system and the analysis of its activity. Gene 647:129-135. 10.1016/j.gene.2018.01.026

Zhang XW, Gureasko J, Shen K, Cole PA, and Kuriyan J. 2006. An allosteric mechanism for activation of the kinase domain of epidermal growth factor receptor. Cell 125:1137-1149. 10.1016/j.cell.2006.05.013 


\section{Figure 1}

FGFR1C kinase (FGFR1K) and phosphorylation sites.

(A) FGFR1c consists of three domains: extracellular receptor domain (D1, D2, D3), transmembrane domain (TM) and kinase domain ( $\mathrm{N}$ lobe and $\mathrm{C}$ lobe). The kinase domain posesses 7 tyrosine autophosphorylation sites, including Y653 and Y654 in the active loop (A-loop). The kinase domain (residues 458-765; FGFR1K) were constructed and recombined expressed for functional studies. (B) Surface representation of inactive (left, PDB ID: 3KY2) and active (right, PDB ID: 3GQI) conformations of the FGFR1 kinase domain. A-loop is shown as a cartoon representation in green, and $Y 653$ and $Y 654$, which are unphosphorylated and phosphorylated in the respective inactive and active forms, are highlighted as yellow sticks.

\section{A}

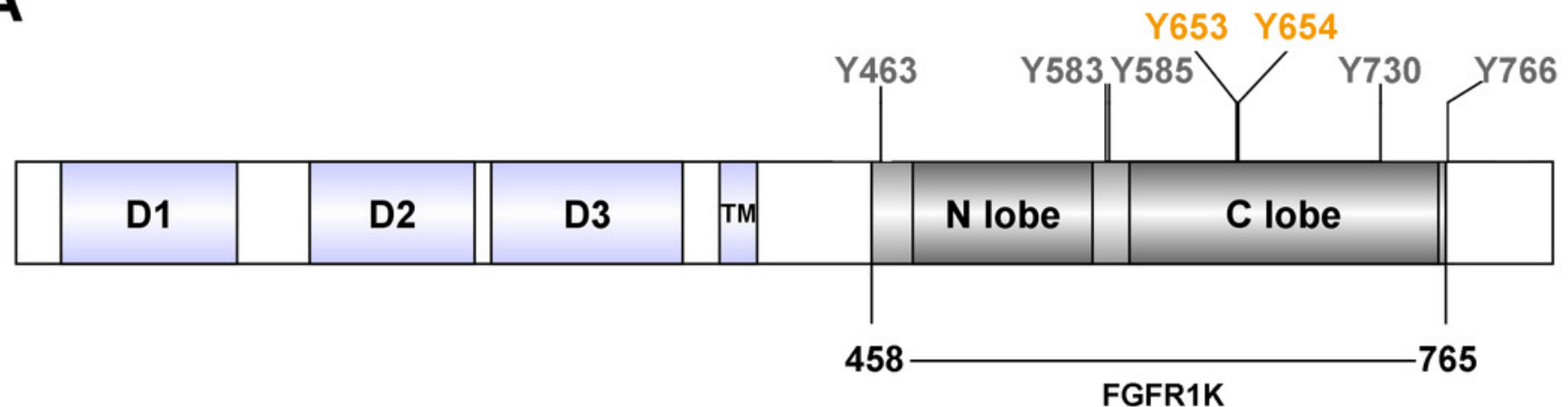

B

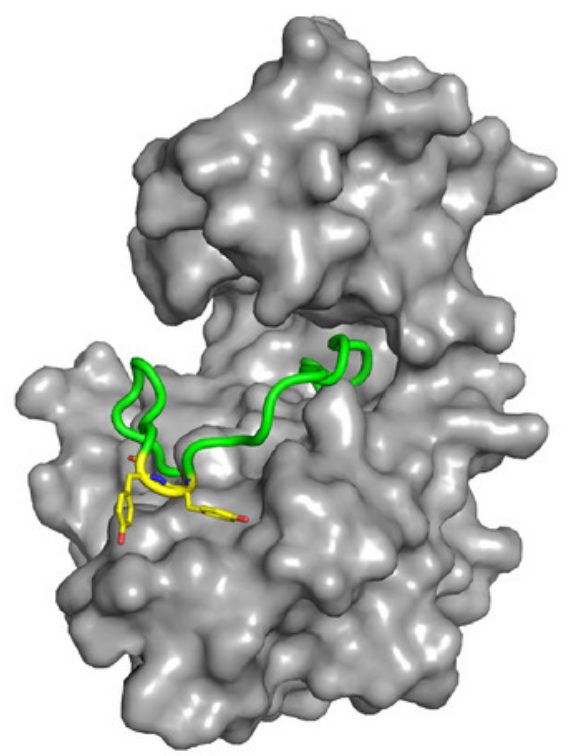

FGFR1K (inactive)

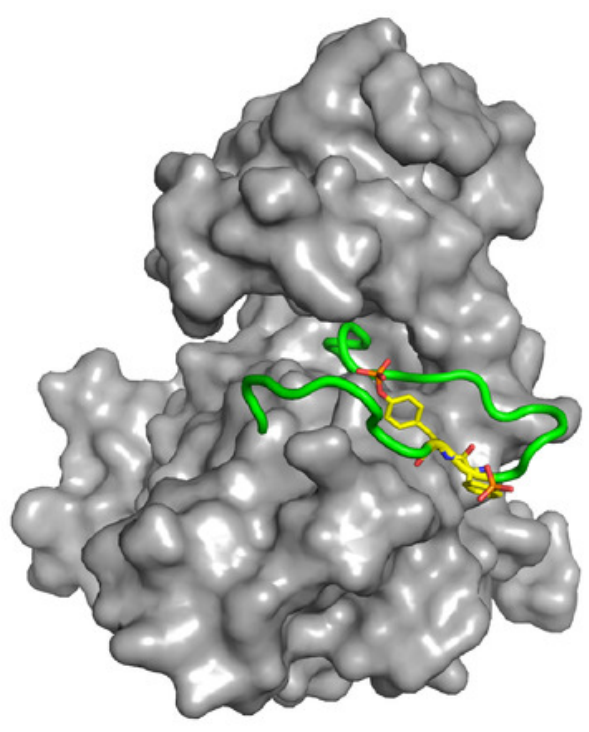

FGFR1K (active) 


\section{Figure 2}

Characterizations of peptide NTA(Ni)-nanodiscs.

(A) Size exclusion chromatography of peptide NTA(Ni)-nanodiscs with lipid:peptide molar ratios of 1:1 (black), 1:3 (red), and 1:9 (blue). (B) Negative stain EM images of 1:1 nanodisc particles ( $12.0 \mathrm{~nm})$. (C) Negative stain EM images of 1:3 nanodisc particles ( $8.6 \mathrm{~nm})$. (D) Negative stain EM images of 1:9 nanodisc particles $(\sim 6.3 \mathrm{~nm})$. The top and side views of nanodiscs were selected from the two-dimensional classified nanoparticles (Supplementary Fig. S2).

A

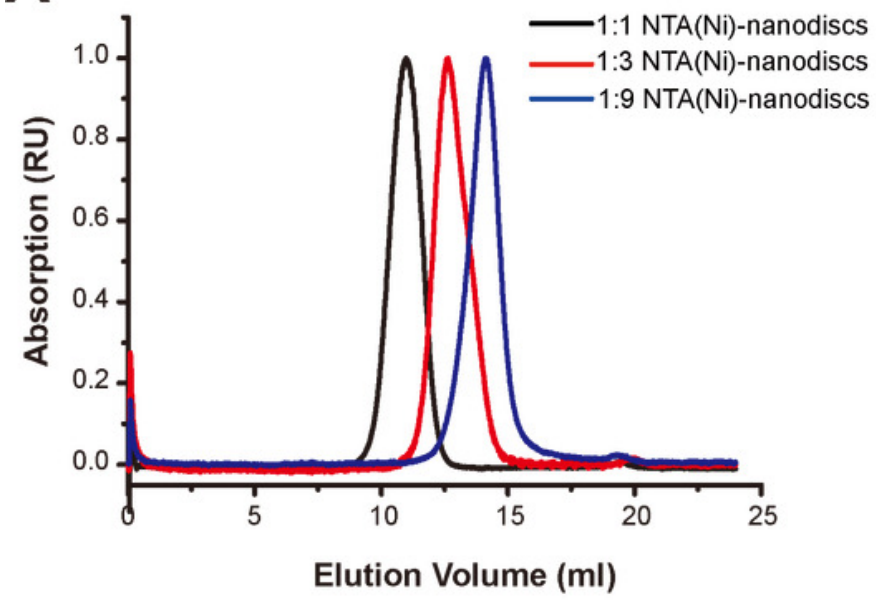

C 1:3 NTA(Ni)-nanodiscs
B

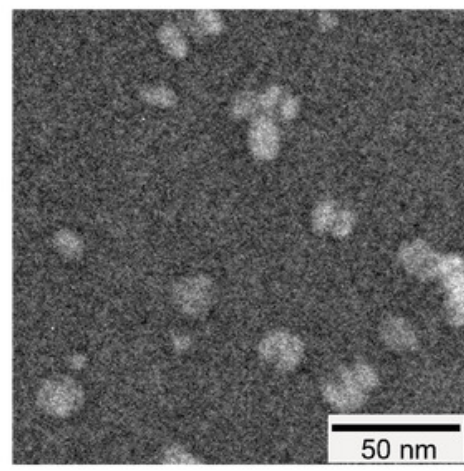

D

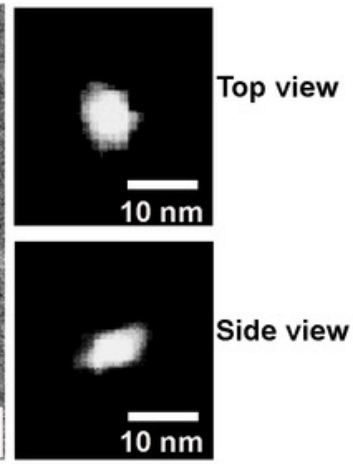

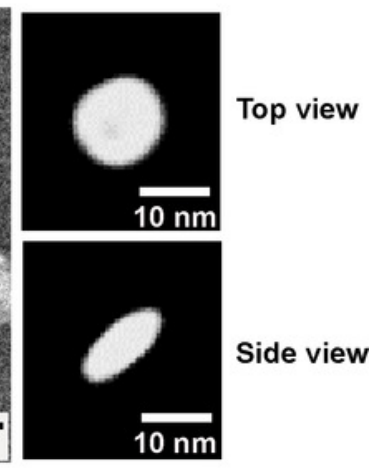
1:9 NTA(Ni)-nanodiscs

\section{1:1 NTA(Ni)-nanodiscs}
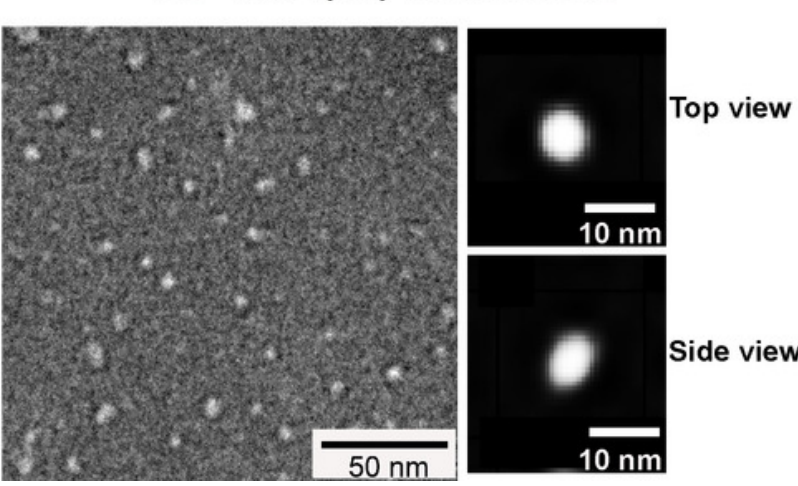

Side view 


\section{Figure 3}

Binding of FGFR1K to NTA(Ni)-nanodiscs.

(A) The size exclusion chromatography of FGFR1K with NTA(Ni)-nanodiscs (1:1) (red line with an elution peak at $10.2 \mathrm{ml}$ ), NTA(Ni)-nanodiscs (1:1) only (black line with an elution peak at $10.9 \mathrm{ml}$ ), and FGFR1K only (blue line with an elution peak at $15.1 \mathrm{ml}$ ). SDS PAGE was performed to verify the formation of FGFR1K- NTA(Ni) nanodisc complex (lane 3), with controls of peptide 22A (lane 1), NTA(Ni)-nanodiscs (lane 2), FGFR1K (lane 4) and protein makers (M). (B) The dissociation constant (KD) of FGFR1K to NTA(Ni)-nanodiscs is determined to be $2.4 \mu \mathrm{M}$ according to the Isothermal titration calorimetry (ITC) measurements. To account for heat of dilution, a control titration of buffer solution into nanodisc solution (Fig. S5) was performed and subtracted from the titration of FGFR1K into nanodisc solution. (C) FGFR1K enzyme activities on peptide NTA(Ni)-nanodiscs of varying diameters. The total phosphorylation and phosphorylation levels of tyrosine 653/4 were detected using western blotting analyses with corresponding antibodies after 10-min reactions. The reaction of FGFR1K in solution was set as control. 

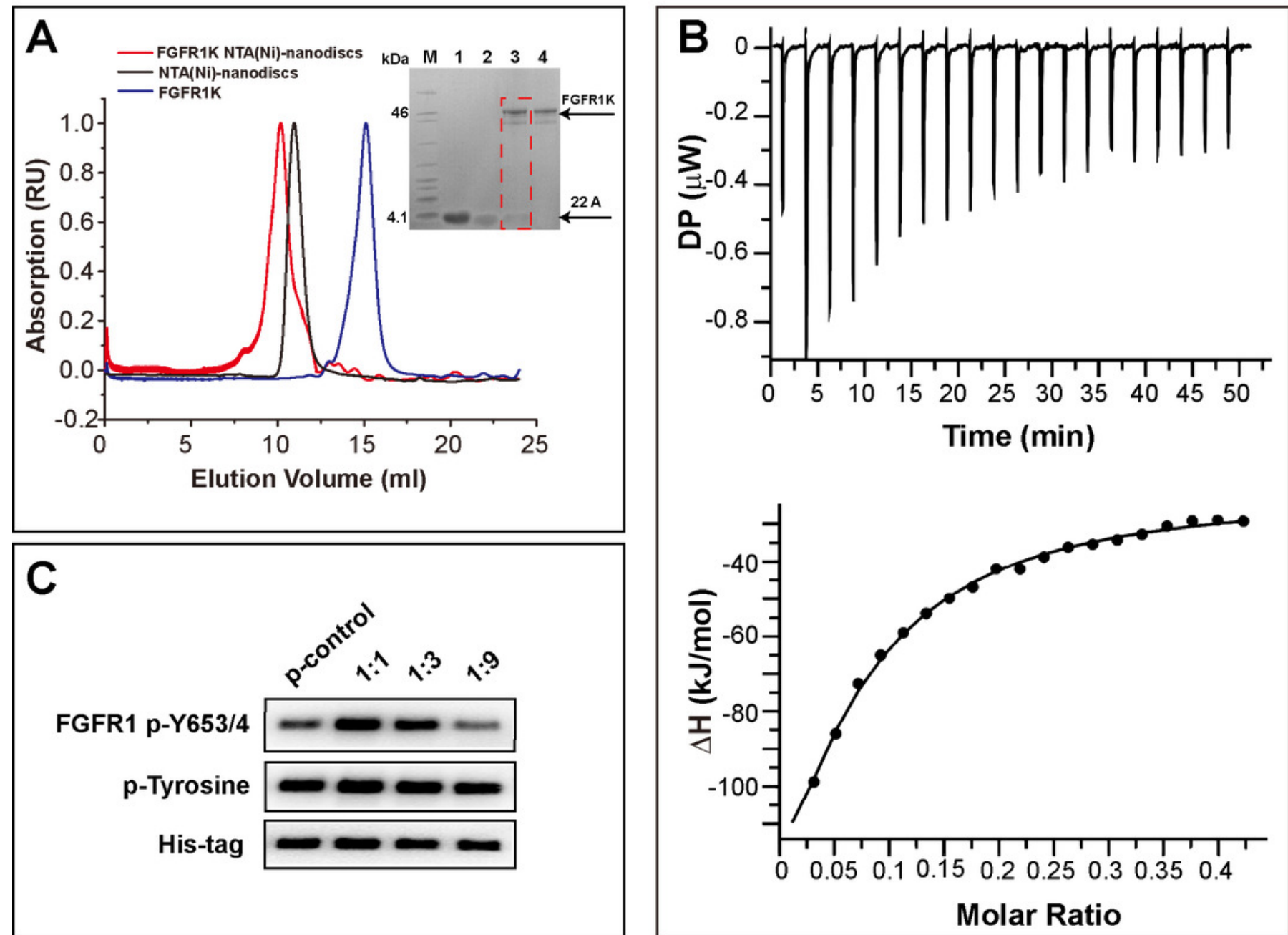


\section{Figure 4}

Autophosphorylation kinetics of FGFR1K in solution and on NTA(Ni)-nanodiscs.

The relative phosphorylation levels of the total tyrosine phosphorylation (A), p-Y653 (B), and p-Y654 (C) of FGFR1K in solution (black lines) and on NTA(Ni)-nanodiscs (red lines) at indicated time points were calculated based on western blotting data (Supplementary Fig. S6). The increment folds of phosphorylation at specific time point is defined by the intensity of the western blotting band relative to that of the starting point. Specific antibodies of phospho-tyrosine, FGFR1 p-Y653, and FGFR1 p-Y654 were used for western blotting analysis. $\mathrm{n}>3 ;{ }^{*} \mathrm{p}<0.05,{ }^{* *} \mathrm{p}<0.01$, and ${ }^{* * *} \mathrm{p}<0.001$. 

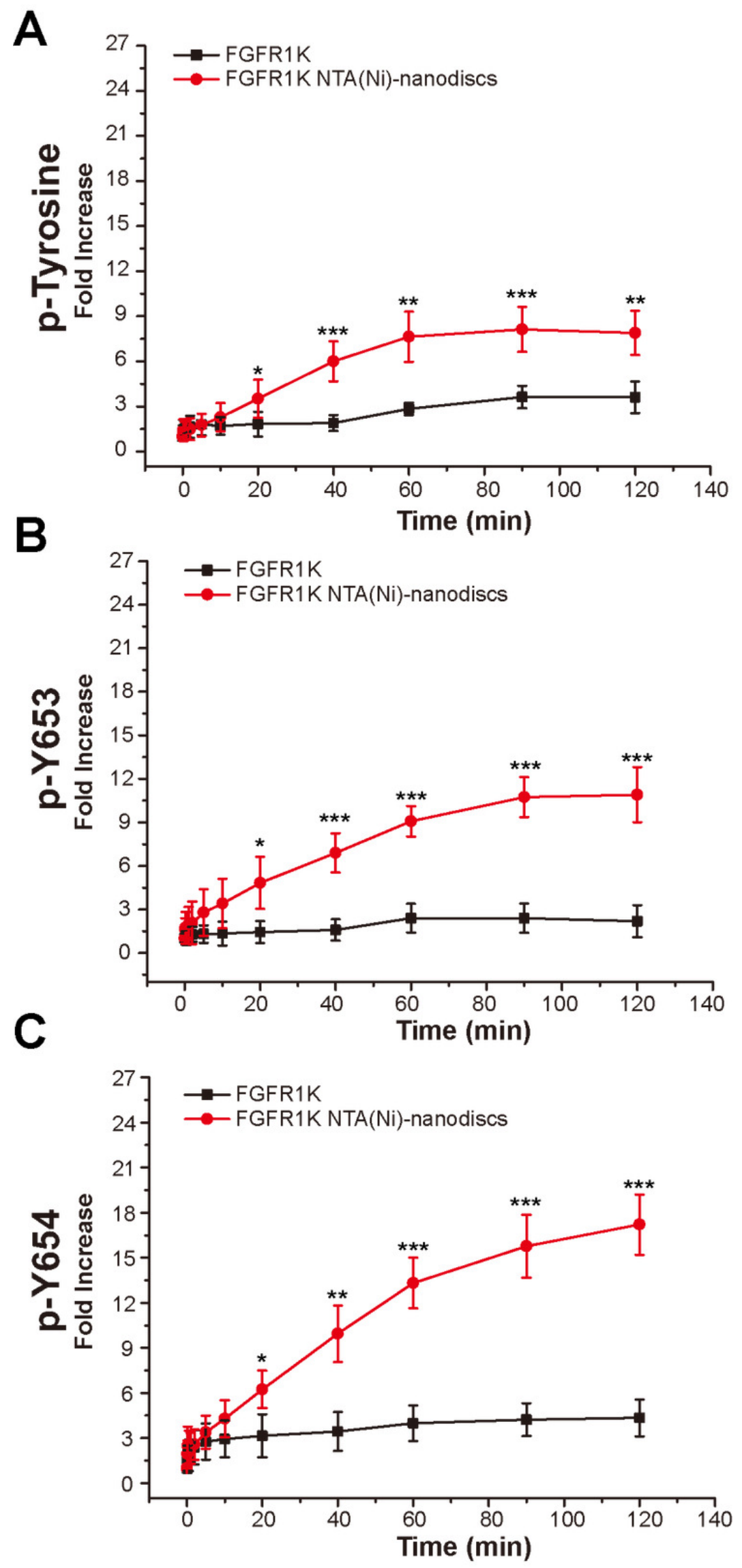